\title{
Macrolide combination therapy for patients hospitalised with community- acquired pneumonia? An individualised approach supported by machine learning
}

\author{
Rainer König (101,2,12, Xueqi Cao ${ }^{1,2,12}$, Marcus Oswald ${ }^{1,2,12}$, Christina Forstner ${ }^{3,4}$, \\ Gernot Rohde ${ }^{5,6,7}$, Jan Rupp ${ }^{6,8}$, Martin Witzenrath ${ }^{6,9}$, Tobias Welte ${ }^{6,10}$, \\ Martin Kolditz ${ }^{11}$ and Mathias Pletz ${ }^{3,6}$, for the CAPNETZ study group' ${ }^{13}$
}

@ERSpublications

A simple decision tree distinguishes patients who benefit from macrolides from those who are harmed. In our model, the rule can lower mortality by $30 \%$ in hospitalised CAP patients with moderate disease. However, prospective evaluation is required. http://bit.ly/2kG5xA5

Cite this article as: König $\mathrm{R}$, Cao X, Oswald $\mathrm{M}$, et al. Macrolide combination therapy for patients hospitalised with community-acquired pneumonia? An individualised approach supported by machine learning. Eur Respir J 2019; 54: 1900824 [https://doi.org/10.1183/13993003.00824-2019].

\section{ABSTRACT}

Background: The role of macrolide/ $\beta$-lactam combination therapy in community-acquired pneumonia (CAP) of moderate severity is a matter of debate. Macrolides expand the coverage to atypical pathogens and attenuate pulmonary inflammation, but have been associated with cardiovascular toxicity and drug interactions. We developed a decision tree based on aetiological and clinical parameters, which are available ex ante to support a personalised decision for or against macrolides for the best clinical outcome of the individual patient.

Methods: We employed machine learning in a cross-validation scheme based on a well-balanced selection of 4898 patients after propensity score matching to data available on admission of 6440 hospitalised patients with moderate severity (non-intensive care unit patients) from the observational, prospective, multinational CAPNETZ study. We aimed to improve the primary outcome of 180 -day survival.

Results: We found a simple decision tree of patient characteristics comprising chronic cardiovascular and chronic respiratory comorbidities as well as leukocyte counts in the respiratory secretion at enrolment. Specifically, we found that patients without cardiovascular or patients with respiratory comorbidities and high leukocyte counts in the respiratory secretion benefit from macrolide treatment. Patients identified to be treated in compliance with our treatment suggestion had a lower mortality of $27 \%$ (OR 1.83, 95\% CI $1.48-2.27 ; \mathrm{p}<0.001)$ compared to the observed standard of care.

Conclusion: Stratifying macrolide treatment in patients following a simple treatment rule may lead to considerably reduced mortality in CAP. A future randomised controlled trial confirming our result is necessary before implementing this rule into the clinical routine.

This article has supplementary material available from erj.ersjournals.com

Received: 25 April 2019 | Accepted after revision: 5 Sept 2019

This study is registered at www.drks.de with identifier number DRKS00005274.

Copyright OERS 2019 


\section{Introduction}

Community-acquired pneumonia (CAP) continues to have considerable morbidity and mortality [1-3]. In hospitalised patients with moderate-to-severe CAP, intrahospital mortality reaches 14\% [4]. Prompt initiation of the correct antimicrobial treatment is essential to prevent unnecessary mortality and complications in patients, particularly in the elderly and other at-risk populations [5]. Macrolides are among the most frequently used antibiotics and currently widely used in treating a broad range of common bacterial infections including upper and lower respiratory infections [6]. Macrolides can be very effective, as, besides their primary antibiotic effect targeting atypical pathogens such as Mycoplasma pneumoniae, Legionella spp. and Chlamydia pneumoniae, they may attenuate inflammation $[7,8]$. However, macrolides can be rather ineffective or even detrimental. They may lead to cardiotoxic side-effects in susceptible patients, as several of these agents have been reported to affect arrhythmia-related cardiac effects, including QT interval prolongation, torsades de pointes, ventricular tachycardia and even sudden cardiac death [9]. In addition, they have been associated with serious interactions to co-medication that is used frequently, e.g. statins.

Numerous observational studies, several meta-analyses and two well-designed randomised controlled trials (RCTs) and cluster-RCTs have investigated the role of macrolide/ $\beta$-lactam versus $\beta$-lactam alone in CAP and have revealed conflicting results [10-12]. The RCT by GARIN et al. [12] enrolling 580 hospitalised CAP patients with a pneumonia severity index (PSI) between I and IV and with negative Legionella urine antigen test on admission found that $\beta$-lactam monotherapy was non-inferior regarding the primary end-point of early clinical stability. However, a subgroup analysis showed that patients with higher severity (PSI IV) and patients with Mycoplasma infection identified later had a significant benefit from the macrolide combination. Even if for PSI I-III there was no effect, the odds ratio and 95\% confidence interval was 1.06 $(0.82-1.36)$, meaning that some patients had a benefit, whereas others were harmed. This study nicely illustrates the limitations of such RCTs even for simple questions, since the result of an RCT is based on the average effect that may neither reflect the situation of the individual patient nor the complexity of the underlying factors (e.g. cardiotoxicity versus coverage of atypical pathogens). The resulting uncertainty is reflected by major guidelines suggesting a macrolide/ $\beta$-lactam combination for severely ill patients, but leaving it at the discretion of the treating physician for patients with moderate CAP.

The Competence Network for Community-Acquired Pneumonia (CAPNETZ) is an observational, prospective multinational cohort study [2]. Approximately 12000 patients from five countries with comparable standards of healthcare (Germany, Switzerland, the Netherlands, Austria and Denmark) have been recruited for $>15$ years. The relevant aspects for CAP treatment and management are listed in the CAPNETZ database, including comorbidities and risk factors, clinical check-up results, historical and clinical therapeutic management, pathogen spectrum and resistance, follow-up, etc. [13]. We investigated the data within this database using an ex ante approach. Without any initial hypotheses about specific patient variables to be associated with treatment benefit, we employed maczhine learning and identified a simple decision rule consisting of three parameters characterising the patient on admission.

\section{Methods}

\section{Patient population}

A detailed description of the CAPNETZ methodology is given elsewhere [2]. It is noteworthy that all patients enrolled in the CAPNETZ cohort study had CAP with at least one of the criteria cough, purulent sputum, history of fever or focal chest signs on auscultation. Patients who had been hospitalised during the 28 days preceding the study and patients with severe immunosuppression or with active tuberculosis were excluded. This prospective multicentre study was approved by the ethical review board of each

Affiliations: ${ }^{1}$ Integrated Research and Treatment Center, Center for Sepsis Control and Care (CSCC), Jena University Hospital, Jena, Germany. ${ }^{2}$ Network Modelling, Hans Knöll Institut Jena, Jena, Germany. ${ }^{3}$ Institute of Infectious Diseases and Infection Control, Jena University Hospital, Jena, Germany. ${ }^{4}$ Dept of Medicine I, Division of Infectious Diseases and Tropical Medicine, Medical University of Vienna, Vienna, Austria. ${ }^{5}$ Dept of Respiratory Medicine, Medical Clinic I, Goethe University Hospital, Frankfurt/Main, Germany. ${ }^{6}$ CAPNETZ STIFTUNG, Hannover Medical School, Hannover, Germany. ${ }^{7}$ Biomedical Research in Endstage and Obstructive Lung Disease Hannover (BREATH), Member of the German Center for Lung Research (DZL). ${ }^{8}$ Dept of Infectious Diseases and Microbiology, University-Hospital Schleswig-Holstein/Campus Lübeck, University of Lübeck, Lübeck, Germany. ${ }^{9}$ Division of Pulmonary Inflammation, and Dept of Infectious Diseases and Respiratory Medicine, Charité - Universitätsmedizin Berlin, Freie Universität Berlin, Humboldt-Universität zu Berlin, and Berlin Institute of Health, Berlin, Germany. ${ }^{10}$ Dept of Respiratory Medicine, Hannover Medical School, Hannover, Germany. ${ }^{11}$ Division of Pulmonology, Medical Dept I, University Hospital Carl Gustav Carus, Dresden, Germany. ${ }^{12}$ These authors contributed equally. ${ }^{13} \mathrm{~A}$ list of members of the CAPNETZ study group can be found in the acknowledgements section.

Correspondence: Mathias Pletz, Jena University Hospital, Center for Infectious Diseases and Infection Control, Am Klinikum 1, Jena, 07747, Germany. E-mail: mathias.pletzamed.uni-jena.de 
participating clinical centre (see www.capnetz.de for participating centres). Supplementary figure S1 depicts the flowchart for the selection and numbers of the patients. Patients admitted to the intensive care unit (ICU) at admission or within 3 days after initial treatment were considered as ICU patients. As, according to major evidence-based guidelines, treating ICU patients with a combination therapy of macrolides and $\beta$-lactams is mandatory, they were excluded from our study, and 6440 patients remained.

\section{Data collection}

In CAPNETZ, patient information is collected using standardised internet-based data acquisition sheets from 2MT (Ulm, Germany) [2]. Patients' characteristics describing health conditions are recorded, including smoking history, chronic comorbidities including chronic kidney disease, chronic liver disease, chronic respiratory disease, history of heart insufficiency (herein "heart insufficiency"), other cardiovascular diseases (herein "cardiovascular comorbidity"), cerebrovascular disease, other neurological diseases, diabetes mellitus and former malignancy [2]. Furthermore, data comprising clinical symptoms and signs at the time of admission, observations from radiology, inflammatory parameters and parameters of the severity of the disease were collected. Particularly, treatment information of each patient was recorded in detail, which included antimicrobial pretreatment within 4 weeks prior to inclusion, initial antimicrobial therapy, change of antibiotic treatment and reasons for this change [14]. The CAPNETZ database contained 1190 variables for each patient, out of which we assembled 78 variables for machine learning, available at the time of the initial treatment decision. For the decision trees, scale variables were binarised by binning the patients into 20 equally filled quantiles leading to 19 binary variables detailing whether or not a patient was below the respective quantile. Supplementary figure S2 shows the flowchart and details for the generation of this assembly. Sputum quality was assessed based on a scheme proposed by BARTLETT et al. [15].

\section{Definition of treatment and primary outcome}

The treatment at admission was defined by the initial antimicrobial therapy. Macrolides (clarithromycin $\mathrm{n}=1923$, roxithromycin $\mathrm{n}=733$, azithromycin $\mathrm{n}=101$, erythromycin $\mathrm{n}=54$ and other macrolides $\mathrm{n}=14$; no patients were given telithromycin) administered orally and intravenously were included. If not indicated otherwise, we grouped patients treated with these macrolides and a $\beta$-lactam antibiotic as macrolide (M)-treated and if treated only with a $\beta$-lactam antibiotic as non-macrolide (nM)-treated. If patients were only treated with macrolides, their treatment was denoted as "macrolide monotherapy". Mortality was assessed at days 28, 30 and 180 [16]. Our primary outcome criterion was death within 180 days since inclusion. Patients who were censored before 180 days since inclusion were considered to be alive $(8.0 \%$ of the stratified patient population).

\section{Stratifying the patient population employing propensity score matching}

Since treatment was not applied by randomisation, a propensity score matching was performed to identify a rule based on a balanced cohort. To note, propensity matching excluded survival, allowing the identification of rules containing only a single variable or the rule to always treat with macrolides. Propensity score matching was calculated using MatchIt [17] ( $m e t h o d=$ nearest, distance=logit, discard=both, ratio=1:1, caliper $=0.2$ ). The caliper parameter was chosen according to the optimal value identified by Austin [18] if scale and binary variables are used. All patient variables were matched for all criteria available on admission and reflecting demographics, disease severity, factors known to be of prognostic relevance (comorbidities) and blood gas analysis-derived lab values (sodium, potassium, glucose, lactate) that are usually available on admission. Scale variables and their squares were $\mathrm{z}$-normalised and binary variables were used unchanged $(0,1)$. The result of MatchIt provided a balanced cohort of 2449 M-treated and $2449 \mathrm{nM}$-treated patients. To estimate the balance between treated and non-treated patients after propensity matching, z-differences were calculated for each variable. In well-balanced data, the z-difference should follow a normal distribution (mean zero, $\sigma=1$ ), i.e. $\geqslant 95 \%$ of all observed values are in $(-2 \sigma, 2 \sigma)$ [19]. All results from machine learning were based on the cohort of these propensity-matched patients.

Furthermore, for each leaf of the tree representing our rule, an additional propensity matching was done. By this, balancing of patients, who have been treated in compliance to our rule versus patients who were treated in compliance to our rule was enforced. If not mentioned otherwise, we used this propensity matching for every leaf for all displayed results of our rule. For the leaves of all stumps, we used MatchIt with the option caliper $=0.2$ as described above. For the leaves of the trees (depth 2), this was not necessary. Instead, for a leaf with low patient numbers (approximately $<100$, which occurred often for condition $\delta$, sometimes for condition $\beta$ and rarely for conditions $\alpha$ and $\gamma$ in figure 1), the number of variables exceeded the number of patients, which caused numerical problems. In these cases, we reduced the number of balancing variables to those having the highest $\mathrm{z}$-difference before matching and used the maximal number of variables with which no numerical problems occurred. After removing all patients with missing values (see next section), $\mathrm{n}=2244$ patients were included, and after propensity matching of all 


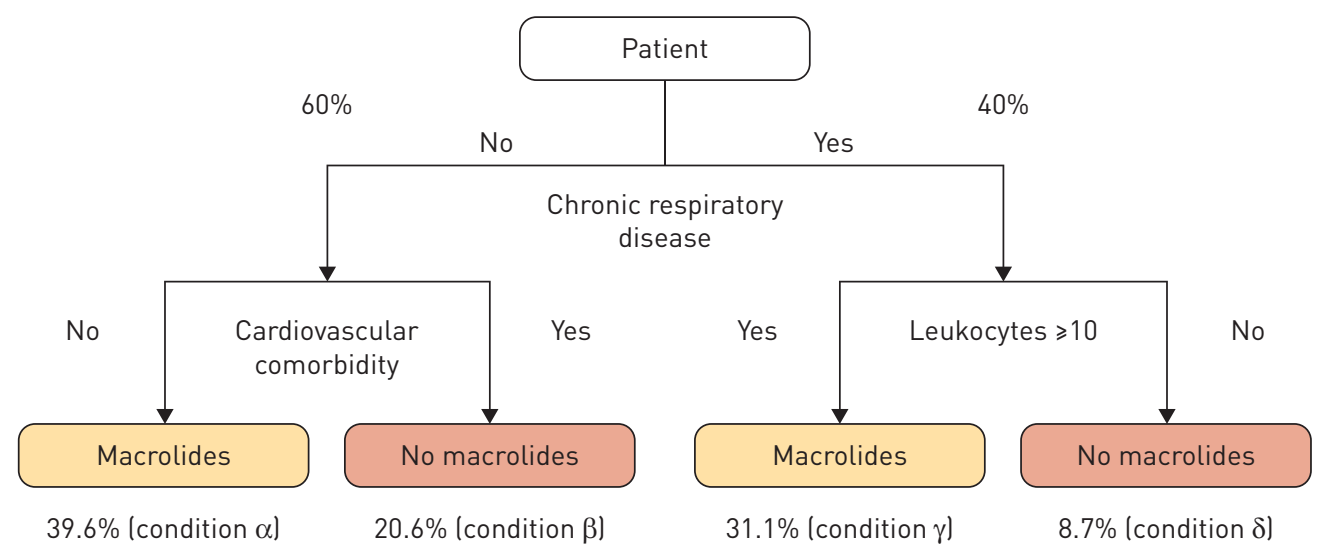

\begin{tabular}{rc|c|c|c|c|c|c|c|} 
& \multicolumn{2}{c}{ Condition $\alpha$} & \multicolumn{2}{c}{ Condition $\beta$} & \multicolumn{2}{c}{ Condition $\gamma$} & \multicolumn{2}{c}{ Condition $\delta$} \\
& Survived & Died & Survived & Died & Survived & Died & Survived & Died \\
\hline M-treated & 354 & 8 & 165 & 23 & 275 & 9 & 69 & 10 \\
\hline nM-treated & 342 & 20 & 170 & 18 & 257 & 27 & 78 & 1
\end{tabular}

In compliance with the rule

Not in compliance with the rule

FIGURE 1 The identified treatment rule depicted as a decision tree. Treating a community-acquired pneumonia (CAP) patient with macrolides is suggested if either 1) the patient has no chronic respiratory disease and no cardiovascular comorbidity (left-hand side) or 2) if the patient has a chronic respiratory disease and shows high or medium leukocyte counts in the respiratory secretion (right-hand side). The rule is now explained for each condition: condition $\alpha: 60 \%$ of the patients $(n=1196)$ had no chronic respiratory disease. Of these, $n=724$ patients had no cardiovascular comorbidity. For these patients, the rule suggests macrolide treatment. In condition $\alpha, n=696$ patients survived if treated according to this rule and $n=28$ died. Condition $\beta$ : if a patient had no chronic respiratory disease, but a cardiovascular comorbidity, the rule suggests no macrolide treatment. This condition was found in $n=376$ patients. In condition $\beta, n=335$ patients survived if treated according to this rule and $n=41$ died. Condition $\gamma: 40 \%$ of the patients $(n=730)$ had a chronic respiratory disease. The rule suggests macrolide treatment, if the leukocyte counts in the respiratory secretion of these patients is medium or high. This condition was found in $\mathrm{n}=568$ patients. In condition $\gamma$, $\mathrm{n}=532$ patients survived if treated according to this rule and $\mathrm{n}=36$ died. Condition $\delta$ : if a patient had a chronic respiratory disease and the leukocyte counts in the respiratory secretion was low, the rule suggests no macrolide treatment. This condition was found in $n=158$ patients. $n=147$ patients survived if treated according to this rule and $n=11$ died. Leukocytes $\geqslant 10$ : leukocytes in the respiratory secretion $\geqslant 10$ per visual field lobject $10 x$, ocular 10x). Leukocytes $\geqslant 10$ is regarded as medium or high. The given percentages refer to the subgroup of the balanced cohort with no missing value in any of the variables being relevant for the respective patient.

leaves, $n=1826$. In particular, for only 2340 patients of the initial 6440 patients the variable leukocyte counts in the respiratory secretion was available. For calculating the performance of our rule when considering the sputum quality, only patients with good sputum quality $(n=1386)$ were selected.

\section{Rationale of the machine-learning approach}

First, we aimed to estimate the impact on the mortality for each single patient variable in CAPNETZ used for the treatment decision to apply combined macrolide treatment. For this, we tested all variables directly as a rule for macrolide treatment. Patients with missing values for the tested variable were removed. For each scale variable, every of the 20 split points from binarisation (see data collection section) was tested and the one with the optimal odds ratio selected. Supplementary figure S2 depicts the flowchart of the assembly of the patient characteristics. For each treatment rule, the improvement of survival was estimated by the odds ratio and reduced death rate. The death rate in a subgroup was calculated by the number of nonsurvivors divided by the total number of patients in the subgroup. To note, this analysis was not based on a cross-validation scheme, and hence these odds ratios may be overestimated. To identify a more effective, but still robust rule, we combined a maximum of three variables in a decision tree of depths two containing a maximum of three nodes. A prototype of such a tree is sketched in supplementary figure S3a. We calculated the odds ratio for every possible combination of variables for such a tree. Supplementary 
figure S3b illustrates such combinations exemplarily for three binary variables. To note, the total number of combinations for such trees is about $n=4 \times 10^{8}$, but by using intelligent enumeration the best tree for a given set of patient samples can be identified in reasonable computational time. To overcome overfitting, we applied a cross-validation scheme. After propensity matching of M-treated and nM-treated patients, we partitioned the patient cohort randomly into 100 parts with equal numbers of patients, and selected the best decision tree based on 99 parts. The selected tree was validated by calculating the odds ratio for this tree based on the patients of the remaining partition. This was repeated 100 times considering each part as the validation set. Finally, after assembling the results of all 100 validation sets, patients with at least one missing value in any of the variables which were relevant for the decision were not considered. In addition, for the result, propensity matching was renewed balancing $\mathrm{M}$ - and nM-treated patients separately in every leaf of the tree.

\section{Statistical testing}

For scale variables, statistical testing for differences in the distributions of the patient variables was performed performing a Mann-Whitney test, and for binary variables a Fisher's exact test, followed by multiple testing correction [20]. p-values for the mortality rates were not corrected for multiple testing. $\mathrm{p}$-value and confidence interval calculation of the confusion matrices for the odds ratios were based on the $\mathrm{R}$ function oddsratio from the "fmsb" package. Kaplan-Meier analysis was performed using the $\mathrm{R}$ functions survfit from the package "survival" and ggsurvplot from the package "survminer". For the Cox hazard regression analysis, we used the function coxph of the R package "survival" (default settings). As survival object we used the complete survival information until day 180 .

Odds ratios were calculated to compare the survival rate of patients that were treated according to the rule with patients that were treated against the recommendation of the rule.

\section{Results}

General characteristics of the study population

Between October 2002 and June 2017, a total of 11818 adult patients from 25 clinical centres with proven CAP were enrolled to CAPNETZ. Hospitalised patients not in the ICU and patients who received either a macrolide/ $\beta$-lactam combination therapy or received only a $\beta$-lactam were selected for our study population. In the following, they are denoted as "M-treated" $(n=2777)$ and "nM-treated" $(n=3663)$ (supplementary figure S1). Table 1 shows the averaged values of the most relevant variables, and variables which were significantly different between $\mathrm{M}$-treated and nM-treated patients. In summary, M-treated patients had a better survival, which, in summary, is most likely explained by younger age, fewer comorbidities and a lower CAP severity, rather than the treatment itself.

\section{Balancing the patient cohorts}

In the initial patient cohort, we observed comparably large differences in critical variables between M-treated and nM-treated patients, in particular, age, comorbidities, severity, inflammatory markers and mortality. To identify clinically usable prognostic variables, we adjusted for all variables and assembled a well-balanced cohort of 4898 patients by matching each M-treated patient with an nM-treated patient (1:1) with same propensity in all variables. The variables are listed in supplementary table S1 (from all patients, before balancing) and supplementary table S2 (from the matched patients). Mortality was not part of this list. z-differences between the groups were calculated and the balance estimated by performing a statistical test for each variable. Despite patients were not matched for mortality, the balanced cohort did not reveal a relevant nor significant difference in mortality (see below). The z-differences of $95 \%$ of the listed variables were in the $2 \sigma$ region, indicating that the data were well-balanced (supplementary table S2). This balanced cohort was used in the next sections.

\section{Evaluating each single patient variable as a rule for treatment decision}

We first investigated each individual patient variable to suit as a rule for the decision to apply macrolides as the initial antimicrobial treatment. The best performing variables are shown in table 2. In contrast to the unbalanced cohort, we didn't observe a noteworthy difference in survival between M-treated and nM-treated patients (OR 1.06, 180-day mortality $7.64 \%$ and $8.08 \%$ of $\mathrm{M}$ - treated and nM-treated patients, respectively). The most powerful single variable to identify patients who benefit from macrolide treatment was high or medium leukocyte counts in the respiratory secretion, followed by non-elevated blood pressure, elevated severity score and absence of cardiovascular comorbidities (i.e. coronary heart disease, hypertension, heart valve defect, cardiomyopathy and arrhythmia). It is noteworthy that chronic heart insufficiency, which can exacerbate during pneumonia, is documented additionally as an individual variable in the CAPNETZ electronic case report form. Furthermore, patients with no chronic respiratory diseases, a high leukocyte count in the blood and a higher CRB-65 (confusion, respiratory rate 
TABLE 1 Comparison of clinical characteristics and mortality between hospitalised patients (not in the intensive care unit) treated with macrolides (M-treated, macrolide combination therapy) and not treated with macrolides (nM-treated)

Initial cohort

Balanced cohort ${ }^{\#}$

\begin{tabular}{|c|c|c|c|c|c|}
\hline & & \\
\hline & M-treated patients & nM-treated patients & Significance of the difference (corrected) & M-treated patients & nM-treated patients \\
\hline Subjects $\mathrm{n}$ & 2777 & 3663 & & 2449 & 2449 \\
\hline Age years & $62.6 \pm 18.0$ & $66.7 \pm 17.1$ & $<0.001$ & $63.2 \pm 17.7$ & $63.9 \pm 17.5$ \\
\hline Male & 61.3 & 59.4 & NS & 60.6 & 61.5 \\
\hline Current smoker & 32.2 & 25.1 & $<0.001$ & 30.9 & 29.3 \\
\hline Nursing home resident & 3.85 & 12.5 & $<0.001$ & 4.37 & 4.53 \\
\hline Confusion & 7.30 & 15.7 & $<0.001$ & 7.8 & 7.7 \\
\hline Enteral nutrition & 3.0 & 4.7 & 0.024 & 3.0 & 2.6 \\
\hline Vaccination against influenza within the past 12 months & 38.0 & 43.8 & $<0.001$ & 38.1 & 38.3 \\
\hline \multicolumn{6}{|l|}{ Comorbidities } \\
\hline Former malignancy & 9.9 & 12.0 & NS & 10.0 & 11.0 \\
\hline Chronic respiratory disease" & 36.9 & 39.0 & NS & 37.2 & 37.2 \\
\hline Chronic kidney disease & 11.7 & 13.2 & NS & 11.6 & 11.2 \\
\hline Chronic liver disease & 3.4 & 3.8 & NS & 3.4 & 3.1 \\
\hline Cerebrovascular disease & 7.6 & 17.7 & $<0.001$ & 8.3 & 7.9 \\
\hline Other neurological disease ${ }^{+}$ & 5.5 & 11.2 & $<0.001$ & 6.1 & 6.0 \\
\hline Diabetes mellitus & 20.8 & 21.7 & NS & 20.9 & 20.4 \\
\hline Heart insufficiency & 18.7 & 27.1 & $<0.001$ & 19.8 & 21.5 \\
\hline Other chronic cardiovascular disease $\S^{\S}$ & 38.8 & 40.0 & NS & 38.9 & 38.5 \\
\hline \multicolumn{6}{|l|}{ Symptoms at time of presentation } \\
\hline Cough & 90.4 & 86.8 & $<0.001$ & 89.8 & 89.4 \\
\hline Purulent sputum & 55.5 & 49.9 & $<0.001$ & 45.5 & 47.2 \\
\hline Fever & 33.2 & 32.7 & NS & 32.5 & 32.4 \\
\hline \multicolumn{6}{|l|}{ Clinical observations } \\
\hline Positive focal auscultation sign & 80.3 & 77.5 & NS & 79.8 & 79.7 \\
\hline Oxygen saturation \% & $92.7 \pm 5.0$ & $93.2 \pm 4.0$ & NS & $92.8 \pm 4.9$ & $93.0 \pm 4.3$ \\
\hline \multicolumn{6}{|l|}{ Radiological observations } \\
\hline Multilobular infiltrate & 14.4 & 13.0 & NS & 14.5 & 14.9 \\
\hline Pleural effusion & 20.9 & 19.8 & NS & 20.7 & 20.2 \\
\hline High or medium leukocyte counts in the respiratory secretion & 29.9 & 22.6 & $<0.001$ & 28.2 & 27.7 \\
\hline \multicolumn{6}{|l|}{ Blood parameters and clinical chemistry } \\
\hline $\mathrm{C}$-reactive protein $\mathrm{mg} \cdot \mathrm{L}^{-1}$ & $151 \pm 120$ & $127 \pm 115$ & $<0.001$ & $146 \pm 120$ & $138 \pm 117$ \\
\hline Thrombocytes $\mathrm{g} \cdot \mathrm{L}^{-1}$ & $252 \pm 108$ & $259 \pm 110$ & 0.036 & $254 \pm 110$ & $255 \pm 109$ \\
\hline Urea $\mathrm{mg} \cdot \mathrm{dL}^{-1}$ & $6.94 \pm 5.06$ & $7.61 \pm 5.51$ & $<0.001$ & $7.02 \pm 5.15$ & $6.99 \pm 4.98$ \\
\hline \multicolumn{6}{|l|}{ Severity parameters and mortality } \\
\hline CRB-65 >1.75 & 21.4 & 29.5 & $<0.001$ & 21.8 & 22.2 \\
\hline CRB-65 score ${ }^{f}$ & $0.964 \pm 0.800$ & $1.14 \pm 0.883$ & $<0.001$ & $0.970 \pm 0.805$ & $0.971 \pm 0.803$ \\
\hline CRB score ${ }^{f}$ & $0.423 \pm 0.594$ & $0.506 \pm 0.678$ & 0.0068 & $0.420 \pm 0.595$ & $0.408 \pm 0.592$ \\
\hline 30-day mortality & 3.6 & 5.7 & 0.0087 & 3.8 & 3.4 \\
\hline 180-day mortality & 7.2 & 11.6 & $<0.001$ & 7.6 & 8.1 \\
\hline \multicolumn{6}{|c|}{ 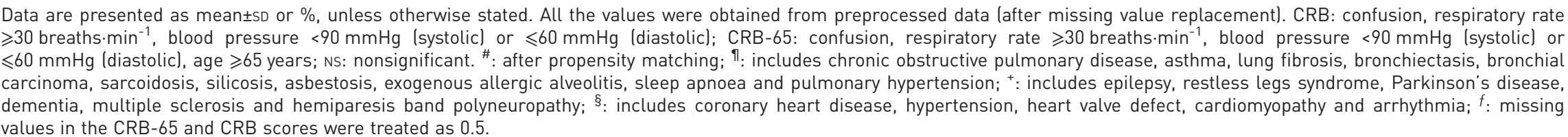 } \\
\hline
\end{tabular}


TABLE 2 Best-performing single variables

\begin{tabular}{|c|c|c|c|c|c|}
\hline & OR $(95 \% \mathrm{CI})$ & p-value & $\begin{array}{l}\text { Compliant } \\
\text { death rate }\end{array}$ & $\begin{array}{l}\text { Non-compliant } \\
\text { death rate }\end{array}$ & $\begin{array}{l}\text { Rate of macrolide } \\
\text { treatment }^{+}\end{array}$ \\
\hline Always apply macrolides & $1.06(0.864-1.31)$ & NS & 0.0764 & 0.0808 & 1 \\
\hline Diastolic blood pressure $<60 \mathrm{mmHg}$ & $1.4(1.14-1.71)$ & 0.0013 & 0.0711 & 0.0965 & 0.212 \\
\hline CRB- $65>1.25^{\S}$ & $1.28(1.03-1.59)$ & 0.025 & 0.0695 & 0.0873 & 0.232 \\
\hline CRB $>0.25^{\S}$ & $1.27(1.03-1.57)$ & 0.027 & 0.0714 & 0.089 & 0.368 \\
\hline $\mathrm{BMI}<29.7 \mathrm{~kg} \cdot \mathrm{m}^{-2}$ & $1.25(1.01-1.54)$ & 0.044 & 0.0704 & 0.0862 & 0.802 \\
\hline \multicolumn{6}{|c|}{$\begin{array}{l}\text { CRB: confusion, respiratory rate } \geqslant 30 \text { breaths } \min ^{-1} \text {, blood pressure }<90 \mathrm{mmHg} \text { (systolic) or } \leqslant 60 \mathrm{mmHg} \text { (diastolic); CRB-65: confusion, } \\
\text { respiratory rate } \geqslant 30 \text { breaths } \min ^{-1}, \text { blood pressure }<90 \mathrm{mmHg} \text { (systolic) or } \leqslant 60 \mathrm{mmHg} \text { (diastolic), age } \geqslant 65 \text { years; BMI: body mass index; NS: } \\
\text { nonsignificant. } \# \text { : death rate of patients who were treated in compliance with the treatment rule; }{ }^{\uparrow}: \text { death rate of patients who were not treated } \\
\text { in compliance with the treatment rule; }{ }^{+}: \text {ratio of patients who were treated with macrolides and who were in compliance with the rule. It } \\
\text { reflects the limitation of the rule to a small group of patients if this rate is far different from } 50 \% ;{ }^{\S}: \text { missing values in the CRB or CRB-65 } \\
\text { score were treated as } 0.5 \text {. Since the thresholds are computed as mean of two consecutive scores, values of a multiple of } 0.25 \text { are possible; } \\
f: \text { other chronic cardiovascular diseases included coronary heart disease, hypertension, heart valve defect, cardiomyopathy and arrhythmia. }\end{array}$} \\
\hline
\end{tabular}

$\geqslant 30$ breaths $\cdot \mathrm{min}^{-1}$, blood pressure $<90 \mathrm{mmHg}$ (systolic) or $\leqslant 60 \mathrm{mmHg}$ (diastolic), age $\geqslant 65$ years) score benefitted from macrolide treatment.

\section{Identifying a decision tree}

To obtain a robust decision rule with an optimal odds ratio, we performed machine learning with level-2 decision trees. Each run of a 100-fold cross-validation resulted in the tree of figure 1 to be optimal with an overall odds ratio of 1.83 , where patients with missing values for respiratory secretion (60\%) where assigned to the "low leukocytes" group. After removing these patients, the overall odds ratio from the cross-validation results increased to OR 2.34 (95\% CI\% 1.56-3.51, $\mathrm{p}<0.001 ; \mathrm{n}=1826$ ). This corresponds to an overall death rate of $3.94 \%$, and a decrease in mortality of $37.9 \%$ of patients identified to be in compliance with the rule compared to the original death rate of $6.35 \%$. Since we had to exclude patients with missing values in any of the three variables, the original death rate of these patients differed from all patients of the balanced cohort in table 1. A Kaplan-Meier analysis accounting for censored data and a time-dependent outcome confirmed the distinct benefit in survival $(p<0.001)$ (supplementary figure S4). Applying a Cox hazard regression model to our rule yielded a hazard ratio of 0.45 (95\% CI $0.31-0.67$, $\mathrm{p}<0.001)$. We investigated whether the sputum quality affected the application of our rule. This was not the case. If we included only patients with good sputum quality, we got comparable results (OR 2.44, 95\% CI 1.51-3.95; $\mathrm{p}<0.001 ; \mathrm{n}=1386)$. Next, we investigated whether the rule was influenced by the detection of an underlying pathogen (either in the blood, sputum, bronchoalveolar lavage, respiratory secretion or aspirate). For these patients, the odds ratio was considerably higher (OR 3.55, 95\% CI 1.57-8.04; $\mathrm{p}=0.001$; $\mathrm{n}=438$ ). In patients in whom a pathogen was either not found or the test was not performed, the odds ratio was lower, but still significant (OR 1.90, 95\% CI 1.14-3.17; $\mathrm{p}=0.013 ; \mathrm{n}=1182$ ). Of note, we observed that M-treated patients showed a tendency of better survival compared to nM-treated patients; however, this was not significant (OR 1.34, 95\% CI 0.920-1.97; nonsignificant).

In condition $(\gamma)$ of figure 1 , patients had a respiratory disease and medium or high leukocyte counts in the respiratory secretion. If, in addition, a patient had a cardiac comorbidity, the rule to treat with macrolides increased the odds ratio for survival considerably (OR 8.01, 95\% CI 1.77-36.3; $\mathrm{p}=0.002 ; \mathrm{n}=194$ ), but note that this amendment to the rule is not based on a cross-validation scheme.

Then, our rule was applied separately to patients treated with macrolide monotherapy and matched controls (treated with $\beta$-lactam). We observed a high odds ratio for patients with macrolide monotherapy, but due to low patient numbers $(n=52)$ this was not significant (OR 4.20, 95\% CI 0.421-41.9). Next, we investigated how the rule performed among subgroups of patients treated with the same macrolides (clarithromycin $\mathrm{n}=613$, roxithromycin $\mathrm{n}=246$, azithromycin $\mathrm{n}=44$ and erythromycin $\mathrm{n}=7$ ) and their propensity matched nM-treated controls. We observed a similar odds ratio for clarithromycin (OR 2.36, 95\% CI 1.46-3.84; p<0.001). For roxithromycin, we observed a lower odds ratio (OR 1.98, 95\% CI 0.8994.34; nonsignificant). A benefit for survival was not significant for roxithromycin; however, this may be not only due to a less favourable efficacy/safety profile, but also the lower number of patients in this subgroup. For erythromycin and azithromycin, the patient numbers were too low to draw any conclusions. 
Odds ratios with confidence intervals and survival rates are given in supplementary table S3a. Supplementary table S3b lists the odds ratios and survival rates for the according patient groups if macrolides were always applied.

As described in the methods section, patients who were admitted to the ICU within the first 3 days after initial treatment were excluded. Including these patients would have misled the machine-learning algorithm, as most of these patients would have received combination therapy even if they were initially treated with a $\beta$-lactam monotherapy. Still, it is reasonable to observe how our rule performed when we included patients who were initially admitted to the regular ward but transferred to the ICU within the next 3 days. However, this didn't change the results (OR 2.24, 95\% CI 1.49-3.37; p<0.001).

In addition, we analysed how the rule performed among patients treated with other antibiotics. Using our rule in patients treated with macrolide $\beta$-lactam combination therapy versus fluoroquinolones (either monotherapy or combined with a $\beta$-lactam) did not reveal significant survival differences. Other comparisons did not yield meaningful results due to low patient numbers.

\section{Discussion}

Testing each patient variable on its own as a single rule, we identified in particular elevated leukocyte count in sputum, but also the following ex ante parameters as variables predicting a benefit from macrolide treatment: absence of cardiovascular comorbidities as well as non-elevated blood pressure and increased CRB-65. The first and the last factors reflect increased inflammation. By testing all combinations of all variables, machine learning came up with the following rule: treat the patient with macrolides if the patient has no chronic respiratory disease and no cardiovascular comorbidity or if the patient has a chronic respiratory disease and shows high or medium leukocyte counts in the respiratory secretion. We rationalise this rule in the following.

Patients with cardiovascular comorbidities including coronary artery disease, hypertension, heart valve defect, cardiomyopathy and prior arrhythmia are at increased risk for macrolide induced QT interval prolongation, ventricular tachycardia, ventricular fibrillation, torsades de pointes and even sudden cardiac death [9, 21-24]. We saw in our data that macrolides had already been intentionally less applied to patients with heart insufficiency. In contrast, such a difference was not observed for patients with the other cardiovascular comorbidities listed above. According to our study, macrolides should be avoided not only in patients with heart insufficiency, but particularly in patients with (other) cardiovascular comorbidities. To note, combining the patient variables heart insufficiency and cardiovascular comorbidities did not improve the performance of our rule. According to our observations, patients with elevated leukocyte counts in blood and the respiratory secretion benefit from macrolide therapy, which may be explained by the known anti-inflammatory effect of macrolides. In line with this, a recent monocentric prospective cohort study [25] revealed that only patients with high inflammatory response and pneumococcal CAP had a benefit from macrolide/ $\beta$-lactam combination therapy (adjusted OR 0.28, 95\% CI 0.09-0.93). To obtain our rule, microbiological aetiology was not considered, since we used only parameters that are available at initial patient assessment. Still, assessing the data from the microbiological diagnosis showed that patients in whom a pathogen was detected would benefit more from the use of our rule than patients in whom no pathogen was detected.

Patients with bronchiolitis obliterans syndrome after lung transplantation particularly benefit from macrolide therapy, if they exhibit an increased bronchoalveolar lavage fluid neutrophil count [26]. A recent systematic review found that the most frequently reported effects of macrolides in clinical studies were a decrease in the number of neutrophils and the concentrations of neutrophil elastase as well as of several pro-inflammatory cytokines [27].

Regarding respiratory comorbidities, the rule partitions the patient cohort into two arms. To get the complete picture, we discuss both arms, as follows. 1) If a patient has neither a chronic respiratory disease nor a cardiovascular comorbidity, then the rule recommends applying macrolides; 2) if a patient has a chronic respiratory disease, macrolides should be applied if leukocyte levels are elevated in the patient's respiratory secretion.

We first discuss arm (1). "Respiratory comorbidities" summarises chronic obstructive pulmonary disease (COPD), bronchial asthma, lung fibrosis, bronchiectasis, former bronchial carcinoma, sarcoidosis, silicosis, asbestosis, exogenous allergic alveolitis, sleep apnoea and pulmonary hypertension. In this arm, patients should be treated with macrolides only if they have neither a chronic respiratory nor a cardiovascular comorbidity, otherwise they should not be treated with macrolides. It is known that COPD can be associated with chronic heart diseases with smoking as a common risk factor [28]. Indeed, in our patients we observed a highly significant correlation of these comorbidities $(\mathrm{p}<0.001$, Fisher's exact test) suggesting that a "chronic respiratory disease" may point to a cardiovascular comorbidity, which may not have been 
detected thus far, accounting for false negatives. Furthermore, patients with both cardiovascular and respiratory comorbidities tend to be older (average age of patients with and without cardiovascular comorbidities 73.3 years and 58.4 years, respectively, $\mathrm{p}<0.001$; average age of patients with and without respiratory comorbidities 68.0 years and 61.5 years, respectively, $\mathrm{p}<0.001$ ) and it is known that the most frequent atypical pathogen, $M$. pneumoniae affects primarily younger patients $[29,30]$. Therefore, patients with these comorbidities may have a reduced benefit, regarding the extended spectrum of macrolides. Regarding arm (2), macrolides are known to attenuate pulmonary inflammation in patients with a variety of lung diseases such as bronchiolitis, cystic fibrosis and non-cystic fibrosis bronchiectasis, and they reduce exacerbation rates in COPD [31,32]. Following our rule in this arm, we suggest that these patients are only treated with macrolides if elevated leukocyte levels are found in the patient's respiratory secretion. Notably, "elevated leukocytes in sputum" was the patient variable with the most impact as a single rule. It is reasonable that macrolides are beneficial when treating an acute respiratory disease with elevated leukocytes levels in the respiratory secretion, as they are known for their anti-inflammatory effects, which are probably conferred by attenuating neutrophil influx into lung parenchyma [7]. Sputum microscopy, which in theory could be performed as point-of-care test, has been considered a standard procedure for many years and was typically performed by microbiologists in order to decide whether the quality of the sample justifies further culture analysis. However, because it is labour-intensive (it requires homogenisation of the sample compared to a simple blood smear [33]) its current use is limited to microbiological laboratories. Therefore, the result may not always be available on the day of admission. Automated devices for point-of-care sputum analysis are currently under development [34].

It is not only important to treat patients with macrolides who will most likely benefit from it, but also to withhold treatment when the risk outweighs the potential benefit. The odds ratio for survival for the rule "always give macrolides" was considerably lower than for our rule. To further explore the underlying mechanism by which macrolides increase mortality, we discuss the patient variable "cardiovascular comorbidity". From a clinical perspective, life-threatening arrhythmias are the most likely cause of macrolide-induced mortality. However, the hazard of a non-observed cardiac arrest due to arrhythmia is highest on the regular ward, where patients are not monitored, in contrast to the ICU. Indeed, we observed a significantly higher death rate of macrolide-treated patients compared to patients without cardiovascular comorbidities at non-ICU units, which we didn't observe for patients at the ICU ( $\mathrm{n}=134$ versus $\mathrm{n}=89$ non-surviving patients at non-ICU units with and without cardiovascular comorbidity, $\mathrm{n}=6$ versus $\mathrm{n}=12$ non-surviving patients at the ICU with and without cardiovascular comorbidity, respectively; $\mathrm{p}=0.025$ employing Fisher's exact test). A smaller fraction of the investigated patients received azithromycin as initial antimicrobial treatment. Azithromycin is one of the novel macrolides and considered as a macrolide with reduced cardiac toxicity and a decreased potential for drug interactions [35]. However, since 2013 the United States Food and Drug Administration have strengthened their warnings and precautions for azithromycin regarding its risk of potentially fatal heart rhythms [21]. Furthermore, RaY et al. [23] showed the cardiotoxic potential of azithromycin in susceptible patients. Excluding these patients didn't change the results (data not shown). In turn, erythromycin and clarithromycin are reported to interact with theophylline, carbamazepine and terfenadine, while there is no similar report for azithromycin [8,36,37]. Considering the distinct profile of azithromycin, it may be studied separately observing a reasonable large patient cohort.

\section{Limitations and strengths}

The limitation of our study is the observational, nom-randomised design. Even if intended, not all consecutive CAP patients are enrolled into CAPNETZ. This may inflict a bias that may reduce external validity. This also applies to the variable "elevated leucocytes in sputum": Even if national recommendations require sputum analysis within $2-4 \mathrm{~h}$ after sampling, the possibility that this time was exceeded for some samples cannot be excluded.

We observed considerable differences in important patient variables of M-treated and nM-treated patients and needed to balance these employing propensity score matching. Hence, to clearly evidence our rule, an RCT is necessary. The strengths of our study are that our analysis bases on a very large and well-phenotyped cohort, and that our analysis focused on simple decision trees employing cross-validation avoiding overfitting, most likely leading to a robust result, and the obtained algorithm is biologically plausible.

\section{Conclusion}

Investigating a well-balanced very large cohort from the CAPNETZ study group employing machine learning, we identified a rule for combined macrolide treatment comprising chronic cardiovascular and chronic respiratory comorbidities as well as leukocyte counts in the respiratory secretion at admission. Since this was not a hypothesis-driven approach, it is very reassuring that machine learning came up with parameters that indeed reflect the advantages and disadvantages of macrolides, i.e. a benefit for patients 
with high pulmonary inflammation and a possible hazard in patients with cardiovascular comorbidities. Clinicians are well aware of macrolide risks and benefits. However, it is difficult to make an optimal decision for an individual patient, in particular with a high inflammatory load, a moderate disease severity and cardiovascular comorbidity, a frequent patient phenotype in CAP. Here the rule helps by prioritising the different decision levels and weighting clearly structured risks against benefits. The rule suggests considerable reduction of the mortality rate 180 days after admission. This rule may be a step towards personalised treatment decisions, but requires proof via RCT.

Acknowledgements: CAPNETZ is a multidisciplinary approach to better understand and treat patients with community-acquired pneumonia. The network has only been made possible by the contribution of many investigators. We are especially indebted to the work of the investigators in the local clinical centres who established and kept contact to all practitioners, physicians, respiratory specialists and all study nurses cooperating within the network.

Members of the CAPNETZ study group are: M. Dreher, C. Cornelissen (Aachen); W. Knüppel (Bad Arolsen); D. Stolz (Basel); N. Suttorp, M. Witzenrath, P. Creutz, A. Mikolajewska (Berlin, Charité); T. Bauer, D. Krieger (Berlin); W. Pankow, D. Thiemig (Berlin-Neukölln); B. Hauptmeier, S. Ewig, D. Wehde (Bochum); M. Prediger, S. Schmager (Cottbus); M. Kolditz, B. Schulte-Hubbert, S. Langner (Dresden); W. Albrich (St Gallen); T. Welte, J. Freise, G. Barten, O. Arenas Toro, M. Nawrocki, J. Naim, M. Witte, W. Kröner, T. Illig, N. Klopp (Hannover); M. Kreuter, F. Herth, S. Hummler (Heidelberg); P. Ravn, A. Vestergaard-Jensen, G. Baunbaek-Knudsen (Hillerød); M. Pletz, C. Kroegel, J. Frosinski, J. Winning, B. Schleenvoigt (Jena); K. Dalhoff, J. Rupp, R. Hörster, D. Drömann (Lübeck); G. Rohde, J. Drijkoningen, D. Braeken (Maastricht); H. Buschmann (Paderborn); T. Schaberg, I. Hering (Rotenburg/Wümme); M. Panning (Freiburg); M. Wallner (Ulm).

Support statement: This work was supported by the project CSCC $(01 \mathrm{EO} 1002,01 \mathrm{EO} 1502)$ of the German Federal Ministry of Education and Research (BMBF), the project CancerTelSys (01ZX1302B, 01ZX1602B) in the e:Med programme and the project KO 3678/5-1 funded by the Deutsche Forschungsgemeinschaft (DFG). The funders had no role in study design, data collection and analysis, decision to publish or preparation of the manuscript. Funding information for this article has been deposited with the Crossref Funder Registry.

Conflict of interest: None declared.

\section{References}

1 Remington LT, Sligl WI. Community-acquired pneumonia. Curr Opin Pulm Med 2014; 20: 215-224.

2 Welte T, Suttorp N, Marre R. CAPNETZ - Community-Acquired Pneumonia Competence Network. Infection 2004; 32: 234-238

3 Almirall J, Bolíbar I, Vidal J, et al. Epidemiology of community-acquired pneumonia in adults: a population-based study. Eur Respir J 2000; 15: 757-763.

4 Ewig S, Bauer T, Richter K, et al. Prediction of in-hospital death from community-acquired pneumonia by varying CRB-age groups. Eur Respir J 2013; 41: 917-922.

5 Welte T. Risk factors and severity scores in hospitalized patients with community-acquired pneumonia: prediction of severity and mortality. Eur J Clin Microbiol Infect Dis 2012; 31: 33-47.

6 Cheng YJ, Nie XY, Chen XM, et al. The role of macrolide antibiotics in increasing cardiovascular risk. J Am Coll Cardiol 2015; 66: 2173-2184.

7 Kanoh S, Rubin BK. Mechanisms of action and clinical application of macrolides as immunomodulatory medications. Clin Microbiol Rev 2010; 23: 590-615.

8 Blondeau JM, DeCarolis E, Metzler KL, et al. The macrolides. Expert Opin Investig Drugs 2002; 11: 189-215.

9 Owens RC, Jr, Nolin TD. Antimicrobial-associated QT interval prolongation: pointes of interest. Clin Infect Dis 2006; 43: 1603-1611.

10 Nie W, Li B, Xiu Q. $\beta$-Lactam/macrolide dual therapy versus $\beta$-lactam monotherapy for the treatment of community-acquired pneumonia in adults: a systematic review and meta-analysis. J Antimicrob Chemother 2014; 69: 1441-1446.

11 Postma DF, van Werkhoven $\mathrm{CH}$, van Elden LJ, et al. Antibiotic treatment strategies for community-acquired pneumonia in adults. N Engl J Med 2015; 372: 1312-1323.

12 Garin N, Genné D, Carballo S, et al. $\beta$-Lactam monotherapy vs $\beta$-lactam-macrolide combination treatment in moderately severe community-acquired pneumonia: a randomized noninferiority trial. JAMA Intern Med 2014; 174: 1894-1901.

13 Suttorp N, Welte T, Marre R, et al. Das Kompetenzzentrum für ambulant erworbene Pneumonie. [CAPNETZ. The competence network for community-acquired pneumonia (CAP)]. Bundesgesundheitsblatt Gesundheitsforschung, Gesundheitsschutz 2016; 59: 475-481.

14 Forstner C, Rohde G, Rupp J, et al. Community-acquired Haemophilus influenzae pneumonia - new insights from the CAPNETZ study. J Infect 2016; 72: 554-563.

15 Bartlett RC. Medical Microbiology: Quality, Cost and Clinical Relevance. New York, John Wiley \& Sons, 1974; pp. 24-31.

16 Zobel K, Martus P, Pletz MW, et al. Interleukin 6, lipopolysaccharide-binding protein and interleukin 10 in the prediction of risk and etiologic patterns in patients with community-acquired pneumonia: results from the German competence network CAPNETZ. BMC Pulm Med 2012; 12: 6.

17 Ho DE, Imai K, King G, et al. MatchIt: nonparametric preprocessing for parametric causal inference. J Stat Soft 2011; 42: 1-28.

18 Austin PC. Optimal caliper widths for propensity-score matching when estimating differences in means and differences in proportions in observational studies. Pharm Stat 2011; 10: 150-161.

19 Kuss O, Blettner M, Börgermann J. Propensity score: an alternative method of analyzing treatment effects. Dtsch Arztebl Int 2016; 113: 597-603. 
Benjamini Y, Hochberg Y. Controlling the false discovery rate: a practical and powerful approach to multiple testing. JR Stat Soc Series B Methodol 1995; 57: 289-300.

21 Albert RK, Schuller JL. Macrolide antibiotics and the risk of cardiac arrhythmias. Am J Respir Crit Care Med 2014; 189: $1173-1180$

22 Bril F, Gonzalez CD, Di Girolamo G. Antimicrobial agents-associated with QT interval prolongation. Curr Drug Saf 2010; 5: 85-92.

23 Ray WA, Murray KT, Hall K, et al. Azithromycin and the risk of cardiovascular death. N Engl J Med 2012; 366: 1881-1890.

24 Hancox JC, Hasnain M, Vieweg WV, et al. Erythromycin, QTc interval prolongation, and torsade de pointes: case reports, major risk factors and illness severity. Ther Adv Infect Dis 2014; 2: 47-59.

25 Ceccato A, Cilloniz C, Martin-Loeches I, et al. Effect of combined $\beta$-lactam/macrolide therapy on mortality according to the microbial etiology and inflammatory status of patients with community-acquired pneumonia. Chest 2019; 155: 795-804.

26 Gottlieb J, Szangolies J, Koehnlein T, et al. Long-term azithromycin for bronchiolitis obliterans syndrome after lung transplantation. Transplantation 2008; 85: 36-41.

27 Zimmermann P, Ziesenitz VC, Curtis N, et al. The immunomodulatory effects of macrolides - a systematic review of the underlying mechanisms. Front Immunol 2018; 9: 302.

28 Morgan AD, Zakeri R, Quint JK. Defining the relationship between COPD and CVD: what are the implications for clinical practice? Ther Adv Respir Dis 2018; 12: 1753465817750524.

29 Dumke R, Schnee C, Pletz MW, et al. Mycoplasma pneumoniae and Chlamydia spp. infection in communityacquired pneumonia, Germany, 2011-2012. Emerging Infect Dis 2015; 21: 426-434.

30 von Baum H, Welte T, Marre R, et al. Mycoplasma pneumoniae pneumonia revisited within the German Competence Network for Community-acquired pneumonia (CAPNETZ). BMC Infect Dis 2009; 9: 62.

31 Naderi N, Assayag D, Mostafavi-Pour-Manshadi SM, et al. Long-term azithromycin therapy to reduce acute exacerbations in patients with severe chronic obstructive pulmonary disease. Respir Med 2018; 138: 129-136.

32 Seemungal TA, Wilkinson TM, Hurst JR, et al. Long-term erythromycin therapy is associated with decreased chronic obstructive pulmonary disease exacerbations. Am J Respir Crit Care Med 2008; 178: 1139-1147.

33 Efthimiadis A, Spanevello A, Hamid Q, et al. Methods of sputum processing for cell counts, immunocytochemistry and in situ hybridisation. Eur Respir J Suppl 2002; 37: 19s-23s.

34 Park HJ, Woo A, Cha JM, et al. Closed-type pre-treatment device for point-of-care testing of sputum. Sci Rep 2018; 8: 16508 .

35 Gorelik E, Masarwa R, Perlman A, et al. Systematic review, meta-analysis, and network meta-analysis of the cardiovascular safety of macrolides. Antimicrob Agents Chemother 2018; 62: e00438-18.

36 Hopkins S. Clinical toleration and safety of azithromycin. Am J Med 1991; 91: 40S-45S.

37 Amsden GW. Erythromycin, clarithromycin, and azithromycin: are the differences real? Clin Ther 1996; 18: 56-72. 